\title{
Simulation-based multi-objective optimization
}

of the product separation process within an MTP plant - Supporting information

Li Zhou, ${ }^{\dagger}$ Zuwei Liao, ${ }^{*} \ddagger$ Lin Wang, ${ }^{\mp}$ Lingling Zhang, ${ }^{\ddagger}$ Xu Ji, ${ }^{\dagger}$ Hongqiao Jiao, Jingdai Wang, ${ }^{\ddagger}$ Yongrong Yang, ${ }^{\ddagger}$ and Yagu Dang ${ }^{\dagger}$

$\dagger$ School of Chemical Engineering, Sichuan University, Chengdu 610065, PR China. $\ddagger$ State Key Laboratory of Chemical Engineering, Department of Chemical and Biological Engineering, Zhejiang University, Hangzhou, Zhejiang 310027, PR China.

\Coal to Liquids Chemical R\&D Center, Shenhua Ningxia Coal Industry Group, Yinchuan 750001, PR China

E-mail: liaozw@zju.edu.cn

Compositions of the input streams of the product separation process The product separation process used in this study consists of two input streams, a liquid hydrocarbon stream and a gaseous hydrocarbon stream. Compositions of the liquid and gaseous streams under designed operation condition are shown in Table S1 
Table S1: Feed compositions for the product separation process of an MTP process.

\begin{tabular}{lll}
\hline \multirow{2}{*}{ Component } & \multicolumn{2}{c}{ Mole fraction } \\
\cline { 2 - 3 } & Liquid stream & Gaseous stream \\
\hline$H_{2}$ & 0.00013 & 0.00892 \\
$C O$ & 0.00081 & 0.02273 \\
$C_{2}$ & 0.00006 & 0.00035 \\
$C_{2} H_{4}$ & 0.01158 & 0.17180 \\
$C_{4} H_{4} O$ & 0.00009 & 0.00074 \\
$D M E$ & 0.00312 & 0.00239 \\
$C_{2} H_{6}$ & 0.01595 & 0.05140 \\
$C_{3} H_{8}$ & 0.00712 & 0.00652 \\
$C_{4} H_{8}$ & 0.12577 & 0.02697 \\
$C_{4} H_{10}$ & 0.10654 & 0.03144 \\
$C_{5} H_{10}$ & 0.08422 & 0.00291 \\
$C_{5} H_{12}$ & 0.11944 & 0.00610 \\
$C_{6} H_{12}$ & 0.03123 & 0.00013 \\
$C_{6} H_{14}$ & 0.11180 & 0.00136 \\
$C_{7} H_{8}$ & 0.00194 & 0 \\
$C_{7} H_{14}$ & 0.12830 & 0 \\
$C_{7} H_{16}$ & 0.00686 & 0.00001 \\
$C_{8} H_{10}$ & 0.00319 & 0 \\
$C_{8} H_{16}$ & 0.00126 & 0 \\
$C_{8} H_{18}$ & 0.00021 & 0 \\
$C_{10} H_{14}$ & 0.00674 & 0 \\
$H C_{O} O C_{2} H_{5}$ & 0.00435 & 0.00028 \\
\hline Flow rate $(\mathrm{kmol} / \mathrm{h})$ & 4655.0 & 937.9 \\
\hline & &
\end{tabular}

\section{Design of algorithm}

Nondominated sorting algorithm (NSGA-II) is applied to solve the multi-objective optimization problem. It is implemented in MATLAB, through which Aspen plus software package is integrated via the Excel-Aspenplus interface for dataexchange. The optimizationalgorithmused in this study is designed as follows, which is also shown in Fig. S1. Step-1,initializethe population for trial solutions with random values distributed within the specified lower and upper bounds, with the predefined population size $P$. Step-2, send the initial value set for the selected decision variablesto Aspen plus for rigorous process simulation, which provides objective values, in this case, purity of the concerned products and the utilitycost, back 
to the main optimizing program through Microsoft Excel. The trial solutions are evaluated to ensure compliance to the problem constraints. The utility costs are calculated in Aspen plus software package by providing the prices and other required parameters. Step-3, sort the population by applying non-domination-sorting method. Rank is assigned based on the objective values of each trial solution. For example, 1 is assigned to the best trial solution without any dominating individuals, and 2 to that of the subsequent and so on. Crowding distance for each trial solution is calculated in the objective space. The trial solutions are sorted based on ascending rank and descending crowding distance. Step-4, select parents among the current generation and reproduce their offspring. Parents of superior quality (the first $P$ trial solutions) are selected based on a tournament selection process which employs the rank and crowding distance as criteria. Genetic algorithm operations, such as simulated binary crossover (with probability equals to 0.9 ) and polynomial mutation (with probability equals to 0.1 ), are used on the previous trial solutions, with step-2 repeated to obtain new trial solutions. Step-5, combine the previous and current population of trial solutions into a combined intermediate population, and sort the intermediate generation by following step-3. Repeat step-4 and onwards till the specified maximum number of generations is meet.

The above algorithm is deployed in MATLAB, through which its conjunction with process simulation in Aspen plus is realized. The optimization is carried out for 10 generations with a population size of 100 , to portray the Pareto-optimal front. Other relevant algorithm parameters used in the optimization are as follows, two-point crossover with probability equals to 0.9 , polynomial mutation with probability equals to 0.143 . More detailed information about the algorithm and its implementation can be found in Deb et al. 1 


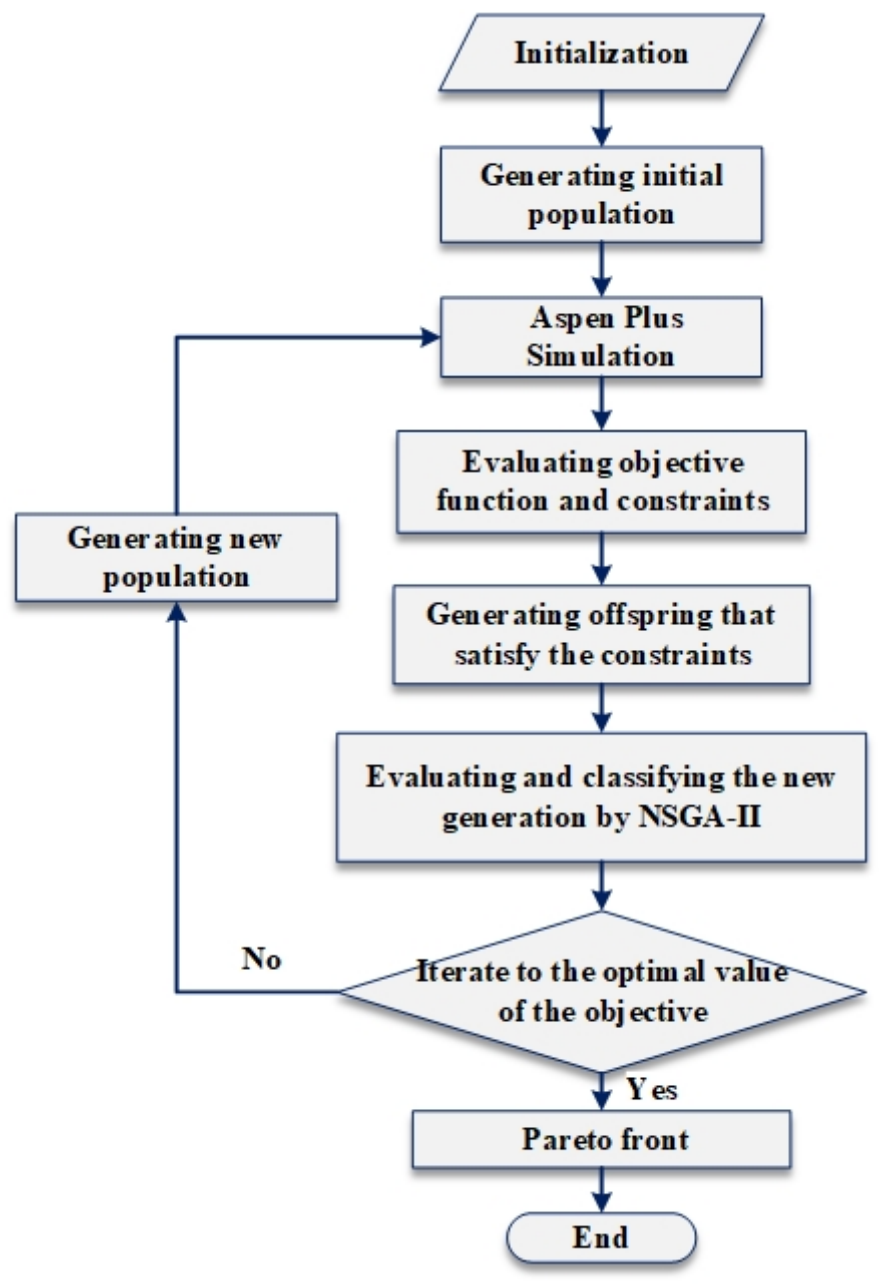

Figure S1: Diagramof the optimizationalgorithm

\section{References}

(1) Deb, K.; Pratap, A.; Agarwal, S.; Meyarivan, T. A fast and elitist multiobjective genetic algorithm: NSGA-II. IEEE Transactions on Evolutionary Computation 2002, 6, 182197. 